\title{
Analytical Model of Fluid Flow through Closed Structures for Vacuum Tube Systems
}

\author{
Chan-Hee Park, ${ }^{1}$ Dae-Sung Cheon, ${ }^{1}$ and Joonam Park ${ }^{2}$ \\ ${ }^{1}$ Korea Institute of Geoscience and Mineral Resources (KIGAM), Daejeon 305-350, Republic of Korea \\ ${ }^{2}$ Wonkwang University, Iksan, Jeonbuk 570-749, Republic of Korea \\ Correspondence should be addressed to Joonam Park; joonam.park@gmail.com
}

Received 25 February 2015; Accepted 30 July 2015

Academic Editor: Stanford Shateyi

Copyright (c) 2015 Chan-Hee Park et al. This is an open access article distributed under the Creative Commons Attribution License, which permits unrestricted use, distribution, and reproduction in any medium, provided the original work is properly cited.

\begin{abstract}
An analytical model is developed to evaluate airtightness, which is one of the most important requirements of vacuum tube transportation systems. The main objective of the model is to anticipate the pressure inside a closed structure, which initially decreases and then rises with time owing to the inflow of the air outside. The model is formulated by using Darcy's law and by solving the differential equations that consider the air permeability of the material and physical configuration of the tube. Equations are derived for a tube section in two cases: one with constant thickness and another with variable thickness. Although the developed model must be verified experimentally, results simulated by using the model with several assumed sections were consistent with the findings of a previous study. The mathematical model developed here to predict the behavior of the pressure inside a vacuum tube structure could be effectively used to evaluate the airtightness of vacuum tube transportation systems. Such data could provide background technical information for the practical design of the system.
\end{abstract}

\section{Introduction}

The air resistance that acts against a transportation vehicle increases with speed. Hence, minimizing air resistance could enhance vehicle speed without excessive consumption of propulsion energy. Vacuum tube systems, which constitute an innovative type of transportation mode where high speeds can be achieved for a vehicle by reducing the air pressure inside a tube structure to minimize air resistance (Figure 1), are increasingly attracting attention for their effectiveness and low emission levels. During the past several decades, transportation systems that use reduced air resistance such as the Vactrain system, Cargo Cap, and PCP (Pneumatic Capsule Pipeline) have been proposed [1-3]. Although these systems differ in their detailed characteristics, they share the basic idea of maintaining the pressure inside a tube structure at a low level. Although an actual vacuum tube system has not yet been realized, according to a recent study [4], the speed of the vehicle could reach up to $700 \mathrm{~km} / \mathrm{h}$ provided that the pressure inside the tube is maintained at $0.1 \mathrm{~atm}$. The system is therefore considered as a strong candidate that could provide an alternative to existing aviation systems.
One of the most important requirements that must be met by the infrastructure of a vacuum tube system is a sufficient level of airtightness. Otherwise, the capacity of the vacuum pumps would need to be inevitably increased, resulting in an increase in construction costs. In addition, the operation interval of the pumps would need to be reduced, increasing maintenance costs as well.

In a vacuum tube, the inside pressure is much lower than the atmospheric pressure outside. Air will then inflow into the vacuum tube structure from the outside unless the tube is completely airtight. The flow rate of air going through a porous medium can be calculated using Darcy's law [5], given the pressure values on both sides. The pressure inside a vacuum tube, however, does not remain constant but increases with time because the tube is a closed hollow structure with finite volume; in contrast, the pressure outside the tube, that is, the atmospheric pressure, remains constant (Figure 2). The pressure inside the tube is therefore treated as a function of time formulated through a differential equation. Mathematical expressions that describe the pressure change inside a vacuum tube with variable thickness are formulated in this study. The differential equation is set up by applying 

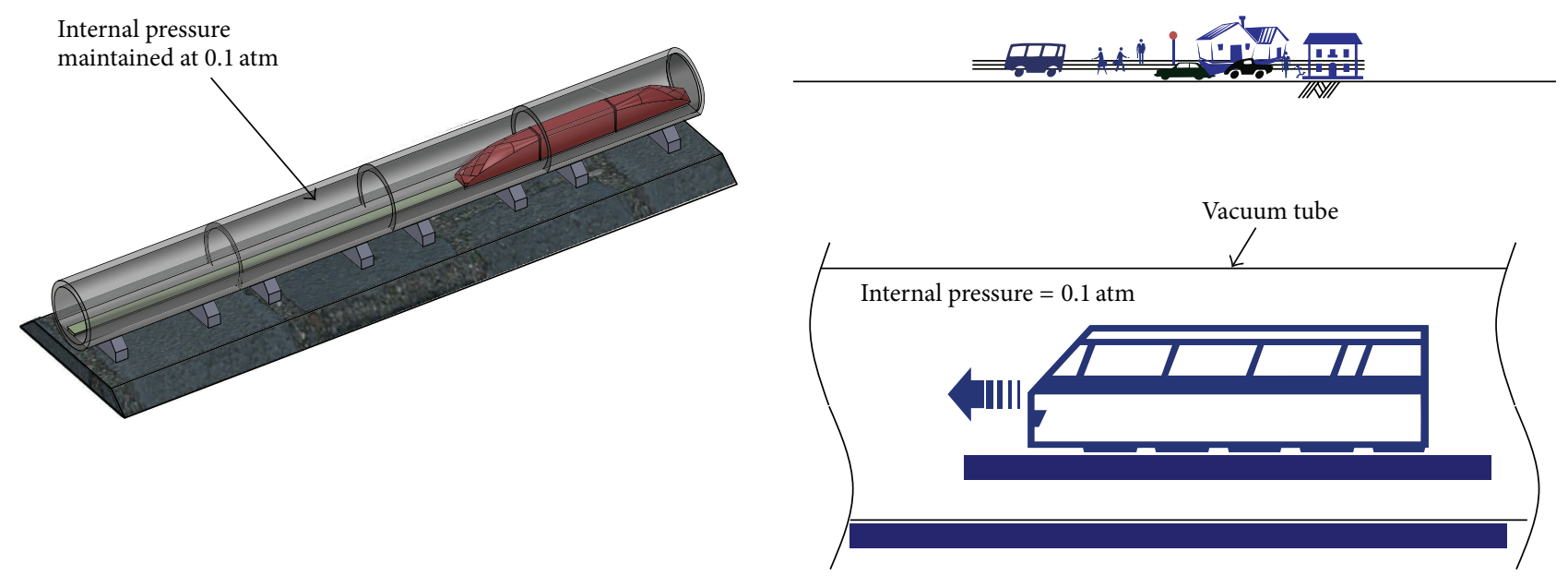

FIGURE 1: Concept of vacuum tube transportation system.
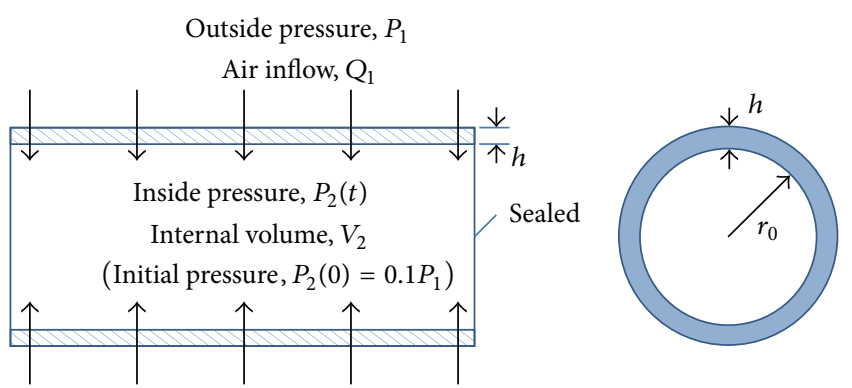

FIGURE 2: Schematic view of a circular vacuum tube with constant thickness.

Darcy's law and considering the physical configuration of the tube structure and initial pressure condition.

\section{Flow Model for Tube Structure with Constant Thickness}

Park et al. [6] have carried out both analytical and experimental studies on evaluating the airtightness of concrete tube structures with different joint configurations. Let us consider a circular tube section with constant thickness as shown in Figure 2, where the initial pressure inside the tube is greatly reduced. As noted above, unlike the pressure outside the tube, which remains constant, the pressure inside the tube increases as air flows into it. Given that the temperature remains constant, the inside pressure increases in proportion to the amount of air inflow. That is,

$$
P_{1} \cdot Q_{1}(t)=\frac{d P_{2}(t)}{d t} \cdot V_{2}
$$

where $Q_{1}$ is the flow rate $\left(\mathrm{m}^{3} / \mathrm{s}\right)$ and $P_{1}$ and $P_{2}$ are the pressures $(\mathrm{Pa})$ outside and inside the tube, respectively.
Meanwhile, the flow rate of compressible fluid that flows into a tube structure can be expressed by applying Darcy's law as follows:

$$
Q_{1}(t)=\frac{k A\left(P_{1}^{2}-P_{2}(t)^{2}\right)}{2 P_{1} \mu h},
$$

where $k$ is the intrinsic permeability of the tube material $\left(\mathrm{m}^{2}\right)$, $\mu$ is the viscosity of the fluid $(\mathrm{kg} / \mathrm{m} \cdot \mathrm{s}), h$ is the thickness of the tube (m), and $A$ is the area across which the air flows per unit length of the tube $\left(\mathrm{m}^{2}\right)$.

Substituting (2) into (1), the gradient of the pressure inside the tube with respect to time is given as follows:

$$
\begin{aligned}
\frac{d P_{2}(t)}{d t} & =\frac{k A\left(P_{1}^{2}-P_{2}(t)^{2}\right)}{2 V_{2} \mu h} \\
& =\frac{k A P_{1}^{2}}{2 V_{2} \mu h}-\frac{k A}{2 V_{2} \mu h} \cdot P_{2}(t)^{2} .
\end{aligned}
$$

By solving the differential equation of form $y^{\prime}=b-a \cdot y^{2}$, the pressure inside the tube is expressed as

$$
P_{2}(t)=P_{1} \cdot \frac{1+C_{1} \cdot \exp \left(-k A P_{1} / \mu h V_{2} \cdot t\right)}{1-C_{1} \cdot \exp \left(-k A P_{1} / \mu h V_{2} \cdot t\right)},
$$

where $C_{1}$ is a constant. The pressure inside the tube when $t=0, P_{2}(0)$, will be the target pressure level for operating the vacuum tube transportation system. $C_{1}$ is determined as $C_{1}=(\beta-1) /(\beta+1)$ when $\beta\left(=P_{2} / P_{1}\right)$ is defined as the initial pressure ratio. If the initial condition is set so that the pressure inside the tube is 10 percent of the outside pressure; that is, $\beta=0.1$; the pressure inside the tube is expressed as

$$
P_{2}(t)=P_{1} \cdot\left[\frac{1-0.8182 \cdot \exp \left(-k A P_{1} / \mu h V_{2} \cdot t\right)}{1+0.8182 \cdot \exp \left(-k A P_{1} / \mu h V_{2} \cdot t\right)}\right]
$$




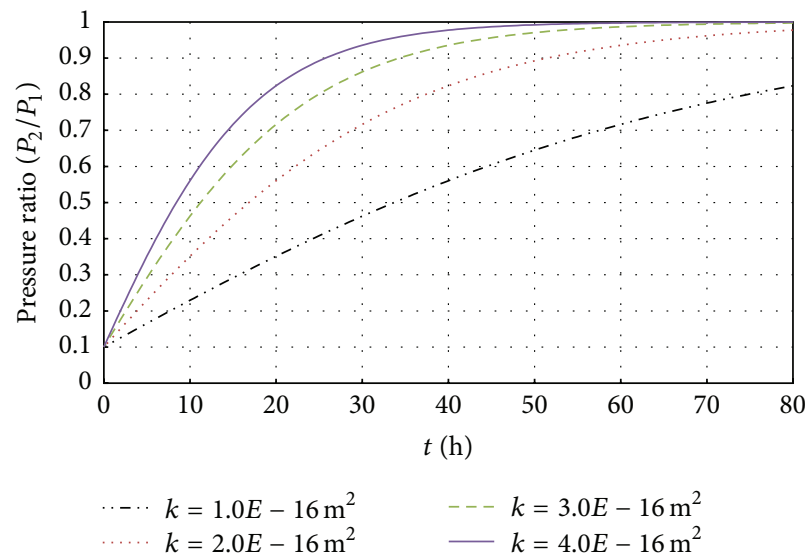

FIGURE 3: Pressure change with time at several levels of air permeability.
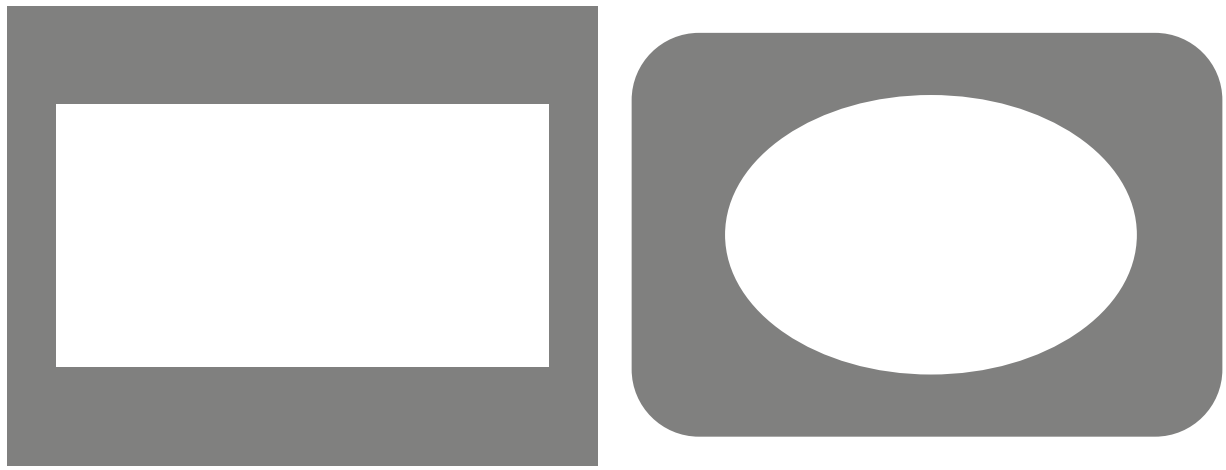

FIgURE 4: Examples of tube sections with variable thickness.

Using (5), the pressure change inside a vacuum tube structure with time can be anticipated. For example, Figure 3 shows the relation between time and the pressure inside a tube with a physical configuration of $r_{0}=1.0 \mathrm{~m}$ and $h=$ $0.15 \mathrm{~m}$ for several different values of intrinsic permeability using (5).

\section{Flow Model for Tube Structure with Variable Thickness}

The mathematical model described in the previous section was established for the special case where the thickness of the tube section was constant. The actual thickness of a vacuum structure, however, may not always be constant; instead, it may vary along the radial direction because a tube section of a structural member is designed in consideration of various loads (e.g., dead, live, and special loads), in addition to cost and constructability (see Figure 4 for examples).

Let us consider a porous medium with a thickness that varies along one direction (the $z$-direction) as shown in Figure 5. That is, the width $b$ and height $l$ of the medium are constant but the thickness $h$ varies along the $z$-direction. Assume that a fluid flow of $Q_{1}$ occurs in the $y$-direction through the medium owing to a pressure drop between $P_{1}$

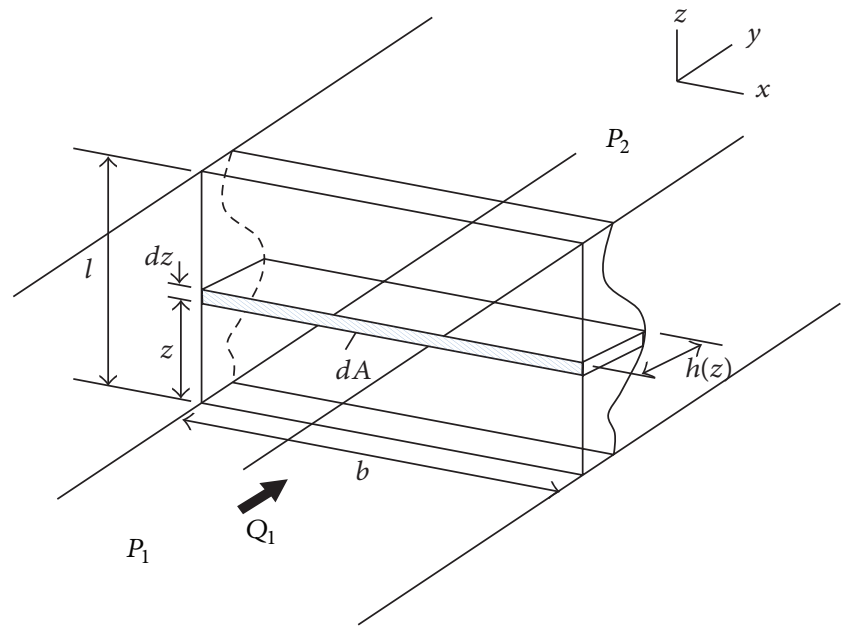

FIGURE 5: Flow of fluid through a porous medium with variable thickness.

and $P_{2}$. The flow rate $d Q_{1}$ corresponding to infinitesimal area $d A=b * d z$ satisfies

$$
d Q_{1}=\frac{k}{\mu h(z)} \cdot \frac{P_{1}^{2}-P_{2}^{2}}{2 P_{1}} \cdot d A .
$$




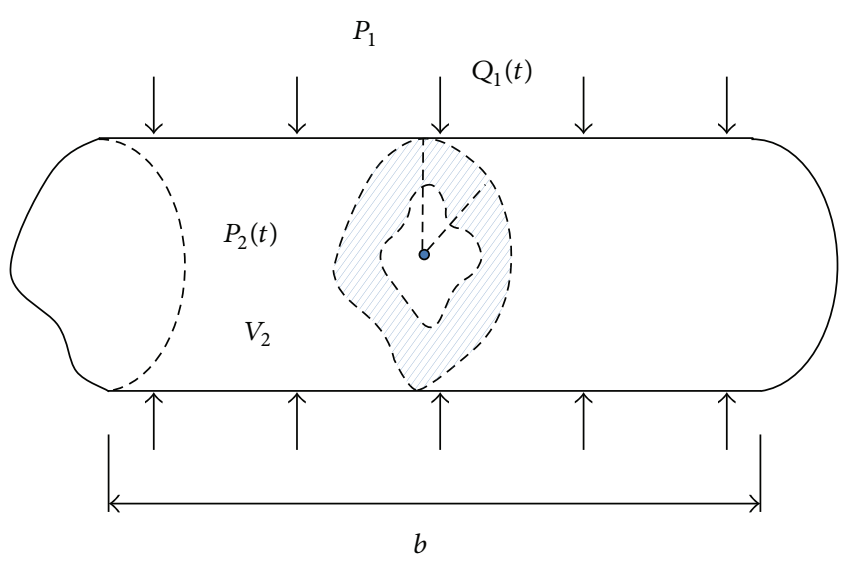

FIGURE 6: Tube structure with variable thickness.

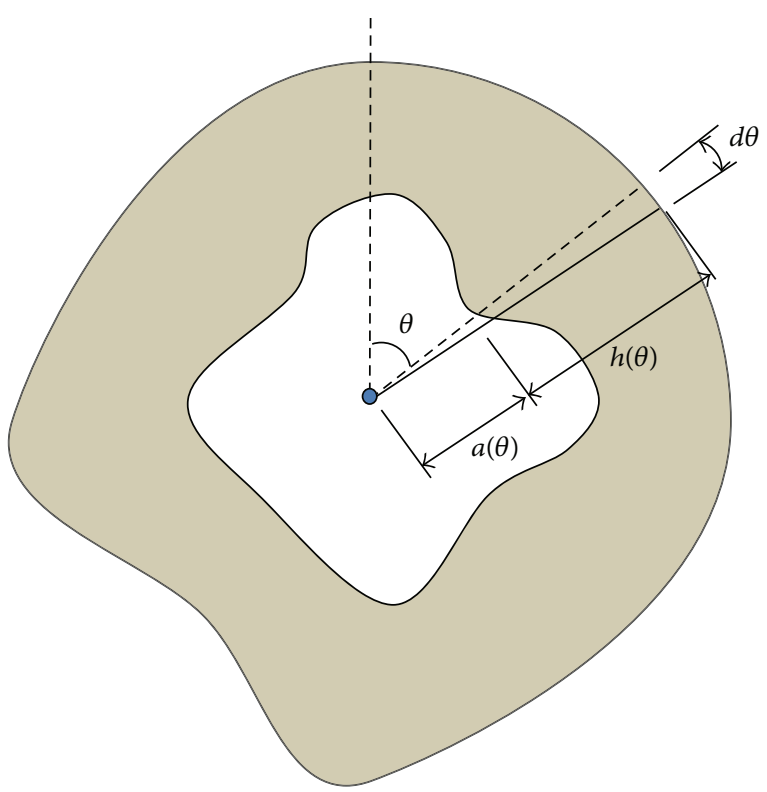

Figure 7: Physical definitions of variables for cross section with variable thickness.

The total flow rate $Q_{1}$ can then be formulated as

$$
Q_{1}=\frac{k}{\mu} \cdot \frac{P_{1}^{2}-P_{2}^{2}}{2 P_{1}} \cdot \int_{0}^{l} \frac{b}{h(z)} d z
$$

Figure 6 shows a schematic view of a tube structure with a length of $b$, of which the thickness is variable along the radial direction. Figure 7 shows a more detailed view of the section where the distances from the centroid to the inner wall and the inner wall to the outer wall are $a(\theta)$ and $h(\theta)$, respectively. The gradient of the pressure change at time $t$ inside the tube is

$$
\begin{aligned}
\frac{d P_{2}(t)}{d t} & =\frac{P_{1}}{V_{2}} Q_{1}(t) \\
& =\frac{P_{1}}{V_{2}} \cdot \frac{k \cdot b}{\mu} \cdot \int_{0}^{2 \pi} \frac{r(\theta)}{h(\theta)} d \theta \cdot \frac{P_{1}^{2}-\left[P_{2}(t)\right]^{2}}{2 P_{1}},
\end{aligned}
$$

where $r(\theta)=a(\theta)+(1 / 2) h(\theta)$.

Upon solving the differential equation, we obtain

$$
P_{2}(t)=P_{1} \cdot \frac{1+C_{1} \cdot \exp (-g \cdot t)}{1-C_{1} \cdot \exp (-g \cdot t)}
$$

where

$$
g=\frac{P_{1}}{V_{2}} \cdot \frac{k \cdot b}{\mu} \cdot \int_{0}^{2 \pi} \frac{r(\theta)}{h(\theta)} d \theta
$$

\section{Illustrative Example}

This section provides illustrative applications of the model developed in this study. Let us first consider a tube section, denoted as Section B, which has a square outer section with a length of $d$ on each side and a circular inner section with a radius $r_{0}$ (Figure 8 ). Assuming that the angle from the vertical line passing through the centroid is $\theta$ and the thickness at that location is $h(\theta)$,

$$
\begin{aligned}
& h(\theta)=\frac{d}{2 \cos \theta}-r_{0} \quad\left(0 \leq \theta \leq \frac{\pi}{4}\right), \\
& r(\theta)=r_{0}+\frac{h(\theta)}{2} \quad\left(0 \leq \theta \leq \frac{\pi}{4}\right) .
\end{aligned}
$$

The pressure inside the tube at time $t$ is then expressed as

$$
P_{2}(t)=P_{1} \cdot \frac{1+C_{1} \cdot \exp \left(-g_{1} \cdot t\right)}{1-C_{1} \cdot \exp \left(-g_{1} \cdot t\right)},
$$

where

$$
\begin{aligned}
g_{1} & =\frac{P_{1}}{V_{2}} \cdot \frac{k \cdot b}{\mu} \cdot \int_{0}^{2 \pi} \frac{r(\theta)}{h(\theta)} d \theta \\
\int_{0}^{2 \pi} \frac{r(\theta)}{h(\theta)} d \theta & =8 \cdot \int_{0}^{\pi / 4} \frac{d+2 r_{0} \cos \theta}{2\left(d-2 r_{0} \cos \theta\right)} d \theta \\
& =\frac{16 d \cdot \ln \left[\left(2 d \cdot \cos (\theta)-4 r+\sin (\theta) \cdot \sqrt{16 r^{2}-4 d^{2}}\right) /(2 d-4 r \cdot \cos (\theta))\right]}{\sqrt{16 r^{2}-4 d^{2}}}-4 \theta .
\end{aligned}
$$




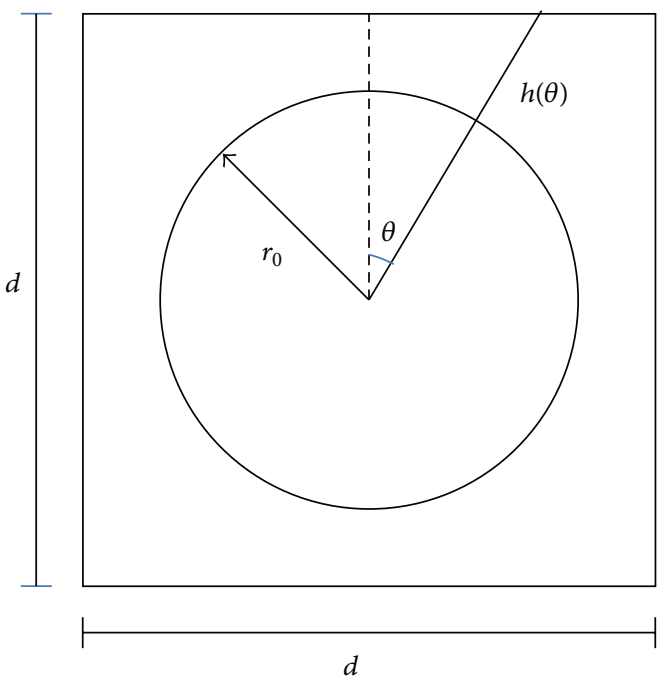

Figure 8: Definition of Section B.

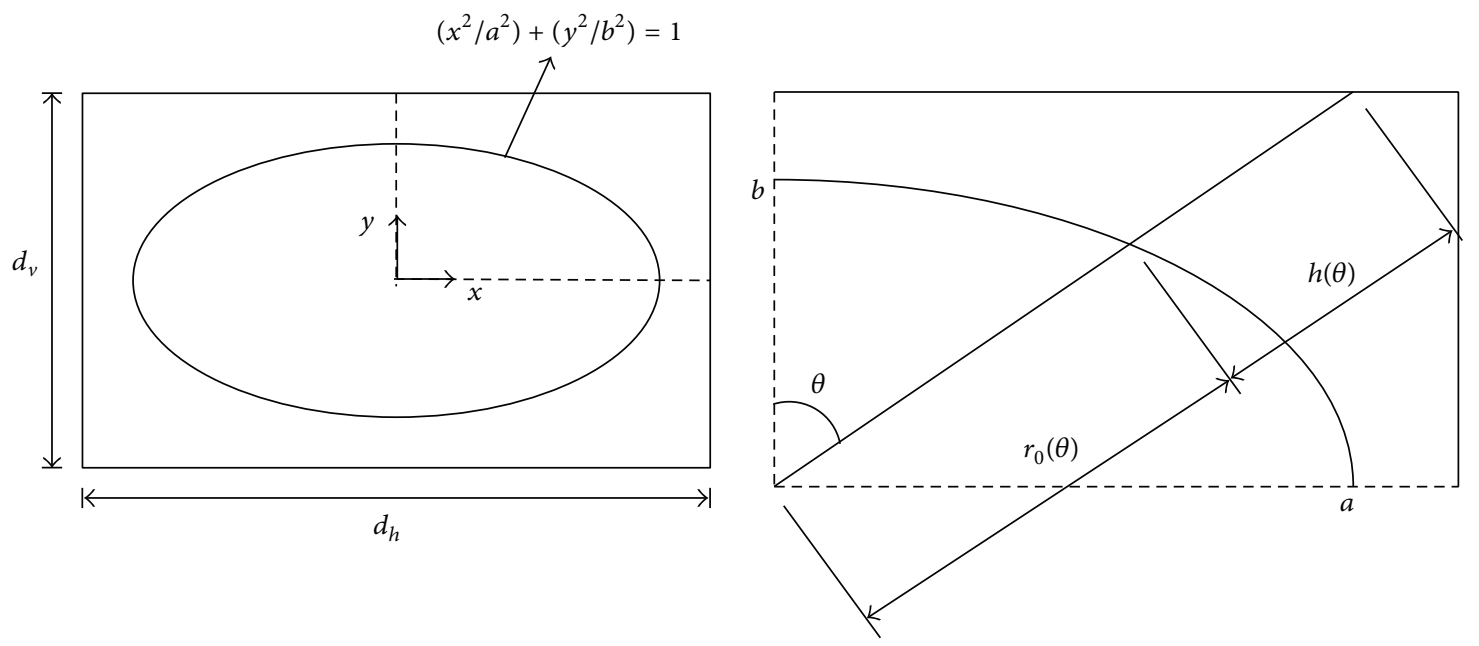

Figure 9: Definition of Section C.

Let us consider one more tube section, denoted as Section $\mathrm{C}$, whose outer section is rectangular and inner section is elliptical (Figure 9). In this case, $r(\theta)$ and $h(\theta)$ are defined as follows:

$$
r_{0}(\theta)=\frac{a \cdot b}{\cos \theta \cdot \sqrt{a^{2}+b^{2} \cdot \tan ^{2}(\theta)}},
$$

$h(\theta)$

$$
\begin{aligned}
= & \begin{cases}\frac{d_{v}-2 r_{0}(\theta) \cdot \cos (\theta)}{2 \cos (\theta)} & 0 \leq \theta \leq \arctan \left(\frac{d_{v}}{d_{h}}\right) \\
\frac{d_{v}-2 r_{0}(\theta) \cdot \sin (\theta)}{2 \sin (\theta)} & \arctan \left(\frac{d_{v}}{d_{h}}\right) \leq \theta \leq \frac{\pi}{2},\end{cases} \\
r(\theta) & =r_{0}(\theta)+\frac{h(\theta)}{2} .
\end{aligned}
$$

Figure 10 shows the pressure change as a function of time for tube structures with three different sections when the intrinsic air permeability is $k=1.0 \times 10^{-16} \mathrm{~m}^{2}$. Section A was a circular section with constant thickness as in Figure 2, with a diameter of $2.0 \mathrm{~m}$ and a thickness of $0.15 \mathrm{~m}$. Section B had a square outer section with a length of $d=2.3 \mathrm{~m}$ on each side and a circular inner section with a radius of $r_{0}=1.0 \mathrm{~m}$. Section $C$ had a rectangular outer section and an elliptical inner section with parameters $a=1.2 \mathrm{~m}, b=1.0 \mathrm{~m}, d_{v}=$ $2.3 \mathrm{~m}$, and $d_{h}=2.7 \mathrm{~m}$ (Figure 9 ). The pressure change plots shown in Figure 10 are for the case where the initial pressure inside the tubes was $10 \%$ of the atmospheric pressure. A comparison of the plots shows that the pressure inside the tube rose most rapidly for the tube with Section A and most slowly for the tube with Section C. This indicates that Sections $\mathrm{C}$ and $\mathrm{A}$ are the most and least airtight, respectively, among the three sections. Note that the average thickness of Section A is smaller than that of the other two sections and that the volume of the space inside the tube is the greatest for Section C. The trend in Figure 10 is therefore consistent with 


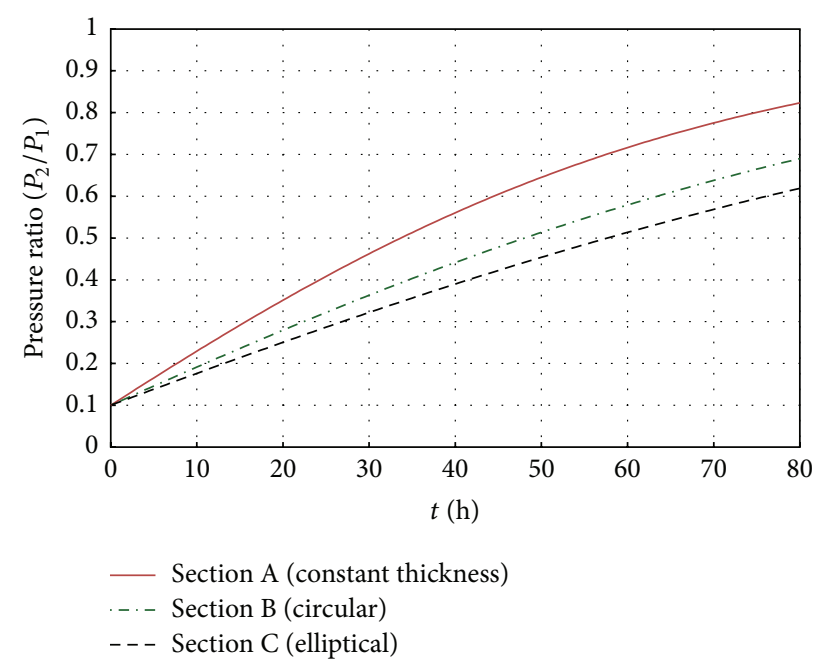

FIGURE 10: Comparison of pressure behaviors for tubes with different sections.

the results of a previous study [6] showing that an increase in the volume or thickness of the tube enhances its airtightness.

Although further experimental verification is needed, the mathematical model developed here to predict the behavior of the pressure inside a vacuum tube structure could be effectively used to evaluate the airtightness of vacuum tube transportation systems. The data thus obtained would provide background technical information for the practical design of the system.

\section{Conclusions}

An analytical model is developed to evaluate airtightness, which is one of the most important requirements of vacuum tube structures. The model anticipates the pressure inside a closed structure, which initially decreases and then rises with time. This is achieved by using Darcy's law and solving differential equations that consider the air permeability of the material and physical configuration of the tube. Equations for the pressure change are formulated for tube sections with uniform thickness and variable thickness. The mathematical model developed is applied to several tube structures with assumed sections. The results show that the tube structure with the largest internal volume is the most airtight. In addition, given the same internal volume, the tube with a section that has a greater average thickness is more airtight. Although further experimental verification is needed, the results simulated by using the developed model are consistent with previous results showing that an increase in the volume or thickness of the tube enhances its airtightness. The mathematical model developed here for predicting the behavior of the pressure inside a vacuum tube structure could be effectively used to evaluate the airtightness of vacuum tube transportation systems. The obtained data could provide background technical information for the practical design of the system.

\section{Disclaimer}

The opinions, findings, conclusions, and recommendations expressed in this paper are those of the authors and do not necessarily reflect those of the sponsor.

\section{Conflict of Interests}

The authors declare that there is no conflict of interests regarding the publication of this paper.

\section{Acknowledgment}

This work was supported by a grant from the International Cooperation R\&D Program of the Korea Institute of Energy Technology Evaluation and Planning (KETEP), funded by the Ministry of Knowledge Economy of the Republic of Korea (2010T100100963).

\section{References}

[1] A. Cassat, V. Bourquin, M. Mossi et al., "SWISSMETROproject development status," in Proceedings of the International Symposium on Speed-up and Service Technology for Railway and Maglev Systems (STECH '03), pp. 453-410, Tokyo, Japan, 2003.

[2] D. A. Becker, "Characterization and use of the new NIST rapid pneumatic tube irradiation facility," Journal of Radioanalytical and Nuclear Chemistry, vol. 233, no. 1-2, pp. 155-160, 1998.

[3] S. K. Choi, "The necessity of development of super speed maglev tube train," Korean Society for Railway, vol. 11, no. 4, pp. 13-18, 2009.

[4] Korea Railroad Research Institute, "Development of new infra-structure technology for ultra high speed tube train (PK09001C)," Project Report, 2009.

[5] P. Mehta and P. Monteiro, Concrete: Microstructure, Properties, and Materials, McGraw-Hill, New York, NY, USA, 2005.

[6] J. Park, L.-H. Kim, S.-W. Nam, and I. Yeo, "Performance evaluation of airtightness in concrete tube structures for superspeed train systems," Magazine of Concrete Research, vol. 65, no. 9, pp. 535-545, 2013. 


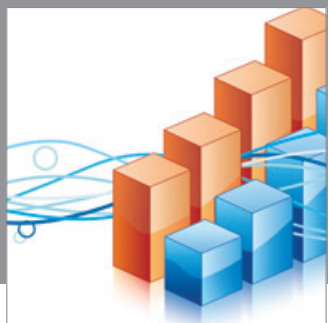

Advances in

Operations Research

mansans

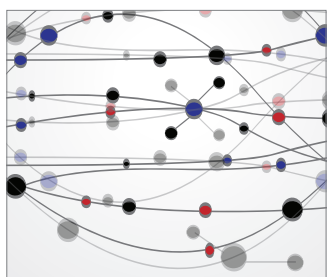

The Scientific World Journal
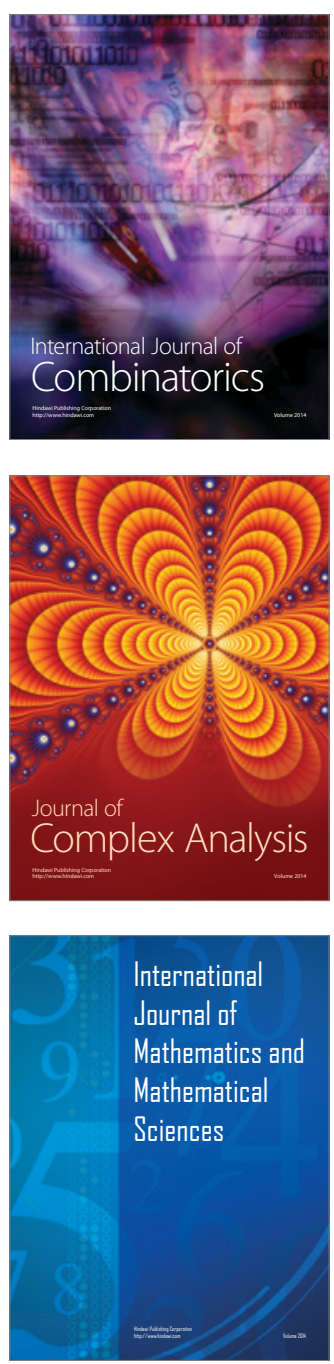
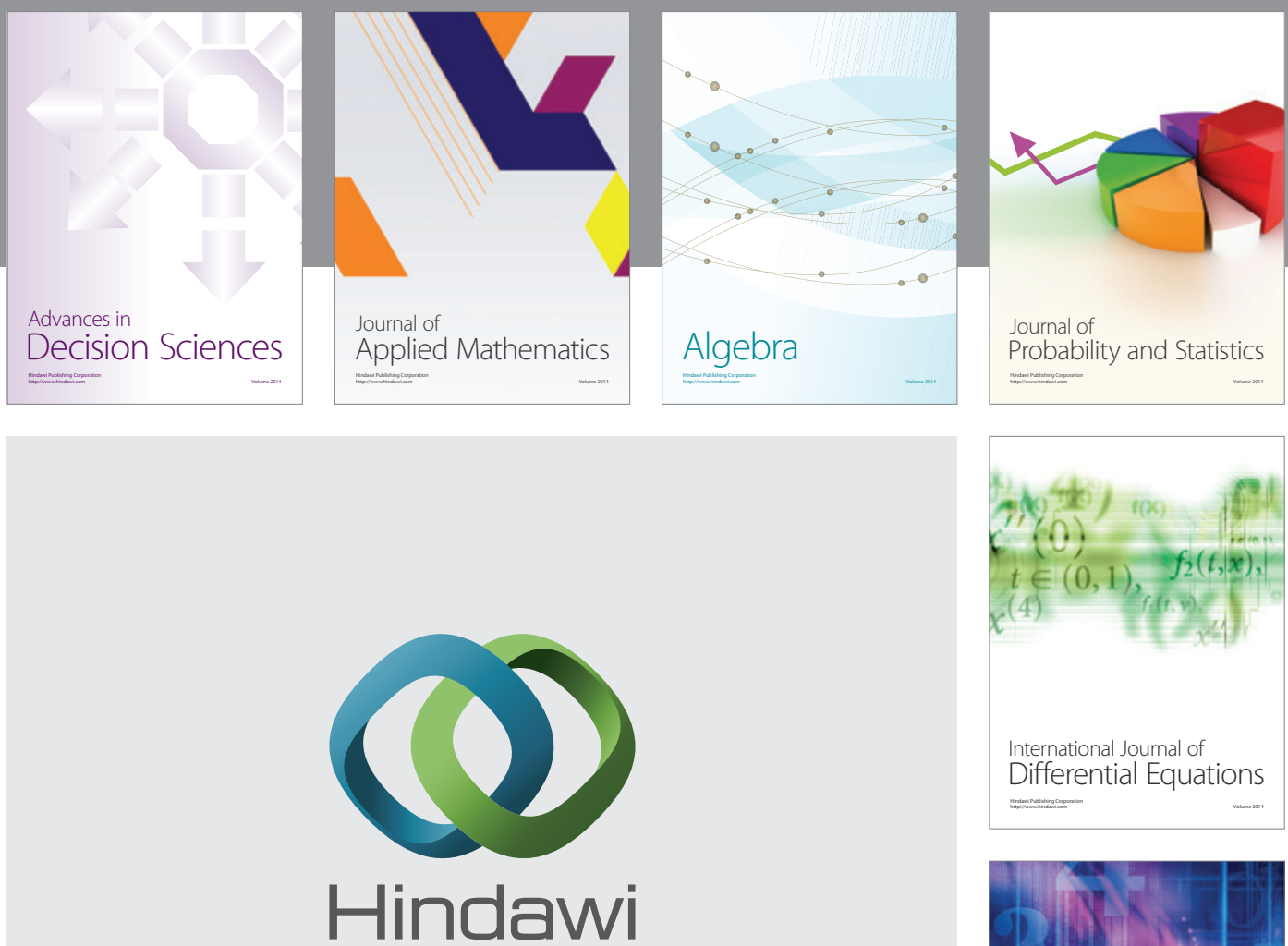

Submit your manuscripts at http://www.hindawi.com
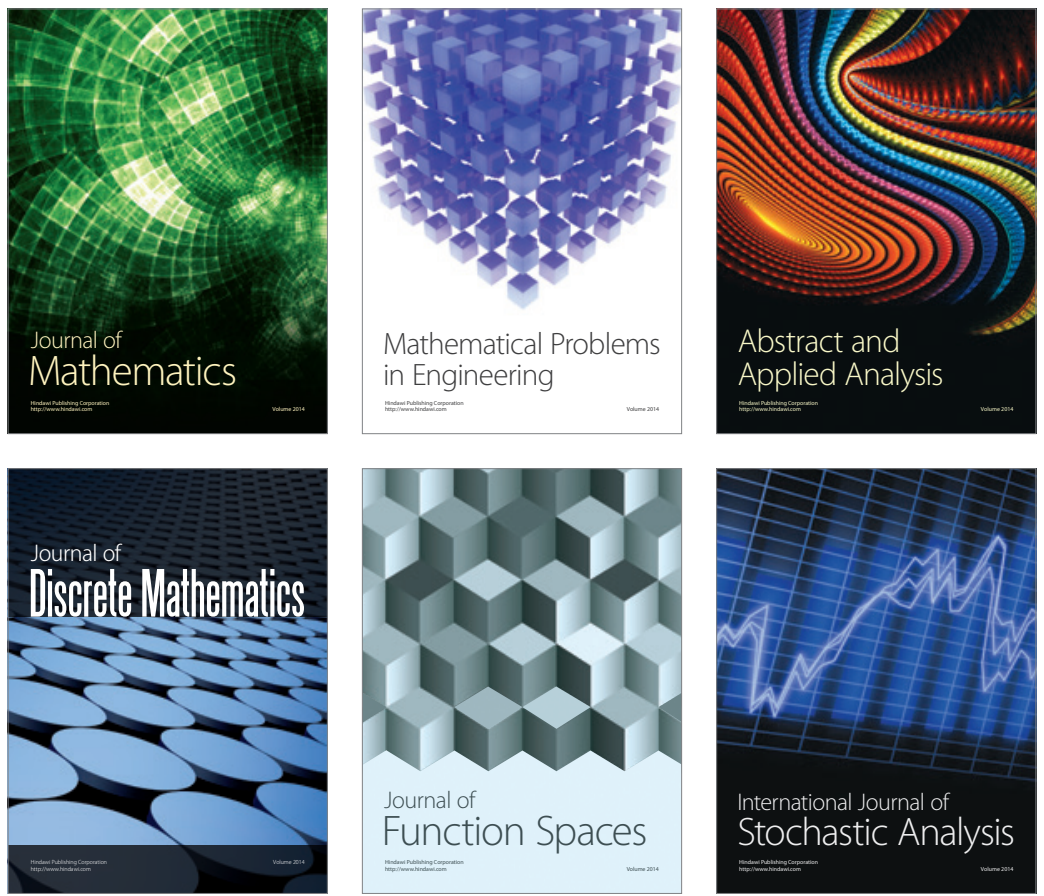

Journal of

Function Spaces

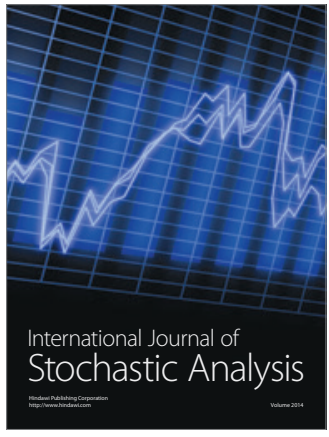

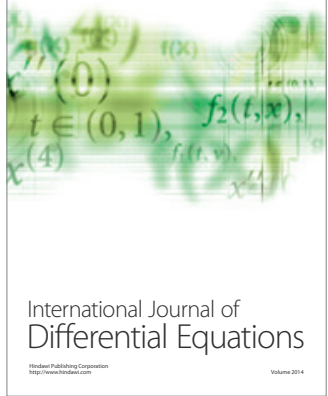
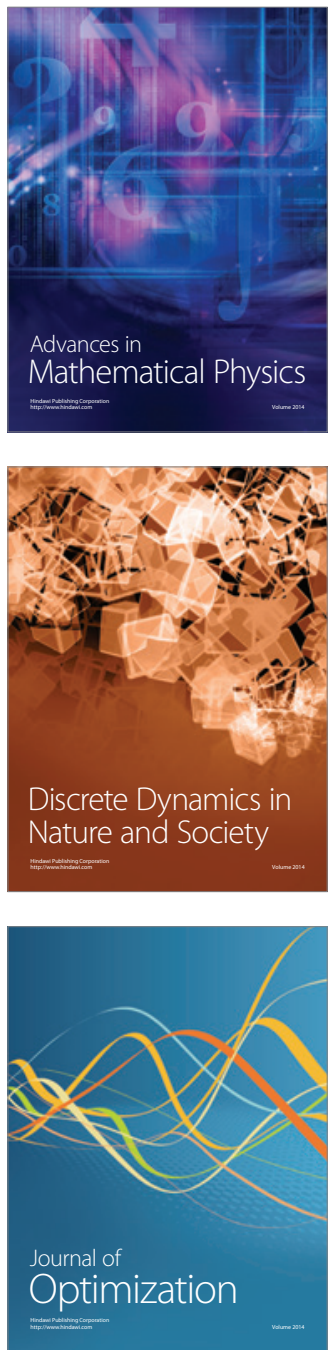